\title{
How are Scottish integrated primary care organisations managed?
}

\section{Steven Simoens and Anthony Scott}

Health Economics Research Unit, University of Aberdeen, Aberdeen, UK

Keywords Primary care, Health care, Organizational structure, Monitoring, Management, Costs

Abstract In the absence of central guidance on the development of integrated primary care organisations, a diversity of models is emerging. This paper examines the management arrangements of Scottish local health care co-operatives (LHCCS). A postal questionnaire survey of all 79 LHCCs was conducted. The response rate was 35 per cent. LHCCs set up management bodies and created workgroups. Stakeholder representation was not socially inclusive: attempts to engage patients and local communities were limited and need to be stepped up to increase responsiveness and accountability to local health care users. LHCCs were also vehicles for local ownership and control of health care provision. To facilitate co-operation among participating practices, LHCCs need to focus on issues of leadership, organisation, and involvement in decision making. Finally, management expenditure per capita was comparable with that of other types of integrated primary care organisations.

\section{Introduction}

The organisational centrepiece of the 1997 reforms of the British National Health Service (NHS) was the establishment of local health care co-operatives (LHCCs) in Scotland and primary care groups/trusts (PCG/Ts) in England (Secretary of State for Health, 1997a,b). LHCCs are voluntary networks of general practitioners (GPs), community nurses, and other health and social care professionals. These integrated primary care organisations co-ordinate the provision of services to, and improve the health of assigned populations. LHCCs are delivering a wide range of primary and community health care services and are involved in cross-sectoral working with local authorities and the voluntary sector. As of 1 May 2000, LHCCs consisted of an average of 12 general practices, $47 \mathrm{GPs}$, and 59,000 people.

An LHCC is an operational unit of a primary care trust (PCT), which is responsible for providing community health services, mental health services, services for people with disabilities, continuing care of elderly people, as well as primary care services based in the general practice. The PCT itself is part of a



This study was funded by the Chief Scientist Office (CSO) of the Scottish Executive Health Department. The authors are indebted to the Information and Statistics Division of NHS Scotland for their co-operation in providing some of the data used in this study. Thanks are also due to Robert McMaster for helpful comments on earlier drafts of this paper and to the LHCCs that participated in the survey. The Health Economics Research Unit is funded by the CSO. The views expressed in this paper are those of the authors and not the CSO.

Journal of Health Organization and Management Vol. 17 No. 1, 2003 pp. $25-36$ (C) MCB UP Limited 1477-7266

DOI 10.1108/14777260310469292 
JHOM

17,1

health board (HB), which is in charge of planning, commissioning, and managing the provision of health care services at regional level.

To date, little is known about how these new organisations in health care operate. Therefore, the aim of this paper is to examine the management arrangements of integrated primary care organisations in the context of LHCCs in Scotland. This is achieved by collecting primary data on the organisational structure of LHCCs, modes of control within LHCCs, relationships among participating practices, and management costs. This is important because the way that integrated primary care organisations are managed may influence the costs and outcomes of health care provision. Finally, implications for the management and operation of LHCCs are discussed.

There are several reasons for examining LHCC management arrangements. Rather than imposing a uniform organisational model, policy makers have allowed the development of LHCCs to be tailored to local circumstances (NHSME, 1999). Hence, there is a need to outline the organisational structure of LHCCs by gathering data about the responsibility and composition of management bodies, range of workgroups, and frequency of management meetings.

There is also a lack of information about how control over and within LHCCs is exercised. Their independence from external actors such as the PCT and HB varies across regions. Moreover, LHCCs are made up of general practices that have no formal line management relationship with LHCC management bodies. To date, LHCC decision making arrangements, including issues such as the involvement of GPs and other health and social care professionals in decision making, management style, and monitoring mechanisms, have not been documented.

The extent to which GPs and other health and social care professionals work together is crucial if LHCCs are to succeed in achieving their goals. "Working together" is also a key issue that underpins the Scottish Health Plan (Scottish Executive, 2000). However, although LHCCs create a structure for integrative processes, this does not guarantee actual co-operation. Thus, it is vital to focus on relationships between participating practices, the factors facilitating and the barriers to co-operation among general practices.

Finally, although the health care reforms announced in the 1997 white paper are expected to generate savings of $£ 100$ million, the introduction of LHCCs is likely to generate additional management costs (Secretary of State for Health, 1997a). This paper reports management allowances allocated to LHCCs and explores the factors that may affect the size of the management allowance.

\section{Research methodology \\ Questionnaire design}

Data were gathered from an anonymous postal questionnaire survey of all 79 Scottish LHCCs. The questionnaire related to the first year of LHCC operation. The design of the questionnaire was informed by a review of the health care 
literature and also benefited from semi-structured interviews with four LHCC general managers. These interviews were conducted between December 1999 and March 2000. The extensive review of the health care literature and expert opinion assured that the questions assessed the desired qualities (face validity) and covered all the relevant domains (content validity).

The questionnaire was based on a number of aspects of the management of integrated primary care organisations that were derived from the economics and health care literature (Simoens and Scott, 1999). Some of these were developed in the context of LHCCs as follows.

The complexity of the LHCC organisational structure was measured by recording the different management bodies and workgroups, their responsibility and composition (Posnett et al., 1998; Regen et al., 1999; Smith et al., 2000).

Different aspects of how control over and within an LHCC is exercised were considered (Grossman and Hart, 1986). The extent to which their development was controlled by PCT management as opposed to driven by the LHCC itself was elicited on a scale of 0 per cent to 100 per cent. A second aspect of the mode of control was the decision making process. The extent to which LHCC managers were involved in decision making compared to GPs and other health care professionals was quantified on a scale of 0 per cent to 100 per cent. Additionally, the management style prevailing in the LHCC was measured on a five-point Likert scale, with a value of one denoting an authoritarian style and a value of five reflecting a fully participative style. Finally, the monitoring process was examined. Respondents had to indicate whether any out of eight possible monitoring mechanisms were used by the LHCC to ensure compliance with its policies.

The relative importance of four types of partnership that can govern the relationship among participating practices was quantified on a scale of 0 per cent to 100 per cent (Pratt et al., 1998). Factors facilitating and barriers to cooperation among participating practices were measured on a five-point Likert scale. Finally, management allowances allocated to LHCCs were used as a proxy of management costs.

The self-administered questionnaire was addressed to the LHCC general manager who would be best placed to know about the issues involved in the development and operation of the LHCC. Information on names and addresses of LHCC general managers was provided by the primary care managers of the 14 Scottish PCTs and by the Information and Statistics Division of NHS Scotland.

\section{Questionnaire piloting}

The questionnaire was piloted in five LHCCs located in Grampian HB, Greater Glasgow HB, and Highlands HB during April-August 2000. An iterative process was set up in which LHCC general managers were asked to discuss the 
JHOM

17,1

28

relevance of the questions, to comment on their wording and interpretation, and to check whether all potential answers were included (face and content validity). Ambiguous questions or answers were deleted or rewritten for greater clarity. Respondents were also asked to fill in the questionnaire. This enabled us to assess the extent of variation in the responses. Comments were elicited during semi-structured interviews. The revised questionnaire was then sent to the general manager of another LHCC. This was repeated until it was felt that only marginal corrections were being proposed.

\section{Statistical analysis}

Comparisons were conducted to investigate whether respondents differed from those that did not respond to the questionnaire in terms of LHCC population and practice characteristics. The one-sample Kolmogorov-Smirnov test was used to check whether continuous variables were normally distributed. Means of variables for the two groups were compared using the two independent samples $t$-test for normally distributed, continuous variables. Medians of variables for the two groups were compared using the two independent samples Mann-Whitney test for not normally distributed, continuous variables.

The results were analysed using simple descriptive statistics. Summary measures were based on the values reported by those who completed the questionnaire. Answers to "yes-no" types of questions were displayed as proportions. The frequency and proportion of LHCCs assigning particular ratings were calculated. Ratings were also averaged across LHCCs and rankings as implied by the order of average ratings were computed. Kendall (1975) argued that these pooled ratings provide the best estimate of the "true" ranking, especially in the absence of any relevant external criterion for ordering objects.

The management allowance was cross-tabulated with population and practice characteristics to assess if there were any patterns by type of LHCC. To test for such associations, two-tailed tests of significance based on the Pearson and Spearman rank-order correlation coefficients were performed, depending on whether variables were normally distributed or not.

LHCC population and practice characteristics included the number of GPs, the number of WTE GPs, the number of general practices, the size of the patient population covered, the number of patients per WTE GP, the age of GPs, the proportion of single-handed practices, the proportion of exfundholding practices, the proportion of training practices, rurality, and population morbidity and deprivation. Rurality was measured by the proportion of people living in urban locations having a population exceeding 500 people. The Arbuthnott index is a composite index of population morbidity and deprivation based on four indicators: the standardised mortality rate among people under the age of 65 years, the unemployment rate, the proportion of elderly people claiming income support, and the number of households with 
two or more indicators of deprivation out of a total of six indicators (Scottish Executive Health Department, 2000).

Data on LHCC population and practice characteristics were taken from a dataset provided by the Information and Statistics Division of NHS Scotland, and the supply and needs database compiled for the National Review of Resource Allocation for NHS Scotland (Scottish Executive Health Department, 2000). All calculations were carried out in SPSS for Windows.

\section{Results}

Response rate

The survey was conducted in November 2000. A first reminder was sent two weeks later and a second reminder was posted three weeks after the first. After two months, 28 questionnaires had been completed and returned, yielding a response rate of 35 per cent.

Respondents and non-respondents were comparable in terms of LHCC population and practice characteristics, except for the proportion of singlehanded practices. Responding LHCCs were more likely to have a higher proportion of single-handed practices $(p<0.01)$. Therefore, data were weighted by the proportion of single-handed practices to ensure that the responding LHCCs used in the analysis would be representative of the population of Scottish LHCCs.

\section{The complexity of the LHCC organisational structure}

The ability of LHCCs to carry out their functions depends on the capacity of professional staff to participate in LHCC management and the development of workgroups. Sixty-four percent of LHCCs adhered to an organisational structure comprising a core group (responsible for strategic management), a management team (responsible for operational management), and a number of workgroups. Three percent of LHCCs had a core group and a management team, but hadn't set up any workgroups. Twenty-four percent of LHCCs were governed by an executive group and covered a number of workgroups. The organisational structure of six percent of LHCCs consisted of an executive group only. Three percent of LHCCs exhibited an elaborate organisational structure including a core group, a co-operative representative council, a management team, and a number of workgroups. A locality approach was adopted by eight percent of LHCCs. This means that groups of general practices existed within the LHCC, each group with their own locality manager.

The most frequently mentioned responsibilities of the core group were concerned with clinical governance, implementation of the LHCC development plan, liaison with other agencies (Acute Trust, social work), service planning, and financial management. Prescribing, representation at PCT/HB level, establishing workgroups, and communication were mentioned less frequently. Core groups on average met every six weeks for about three hours.

Integrated primary care 
JHOM

17,1

The number of people on core groups ranged from eight to 25 , with an average of 15. The LHCC chair (89 per cent of LHCCs), representatives of participating practices ( 82 per cent of LHCCs), other health care professionals, e.g. pharmacists (63 per cent of LHCCs), and professions allied to medicine (59 per cent of LHCCs), were most likely to be involved in the core group. The LHCC general manager and nurse representatives were always members of a core group. Lay representatives, acute sector representatives and voluntary services representatives had joined the core group of only 19 per cent, 13 per cent and 9 per cent of LHCCs, respectively.

Management teams were most likely to be engaged in carrying out support functions (e.g. finance, human resources, infrastructure), day-to-day operational management, implementing LHCC development plans, responsibility over workgroups, and liaison with LHCC members. This was followed by service development, contributing to LHCC development planning, LHCC representation, and providing feedback to and informing the core group. On average, meetings took place every two weeks and lasted around two hours.

Management teams consisted of an average of five people, with a minimum of two and a maximum of seven people. The management team generally included the LHCC general manager (93 per cent of LHCCs), nurse representatives (76 per cent of LHCCs), and LHCC managers (65 per cent of LHCCs). However, mental health representatives, local health council representatives, voluntary services representatives, and lay representatives had not joined the management team of any LHCC.

Executive groups were most likely to deal with clinical governance, service planning, prescribing, financial management, and establishing workgroups. They were also involved in liaison with other agencies (Acute Trust, social work), communication, implementation of an LHCC development plan, day-today operational management, responsibility over workgroups, and carrying out support functions (e.g. finance, human resources, infrastructure). Eight weeks on average elapsed between successive meetings, each of which took around four hours.

The number of people on executive groups ranged from six to 30 , with an average of 15. Although the LHCC chair (100 per cent of LHCCs), nurses (90 per cent of LHCCs), the LHCC general manager (75 per cent of LHCCs), staff of participating practices (65 per cent of LHCCs), and the LHCC vice-chair (54 per cent of LHCCs) were well represented on executive groups, mental health service representatives, voluntary service representatives, and lay representatives were not included in the executive group of any LHCC.

Responsibility for functions or activities was assigned to a specific workgroup in 89 per cent of LHCCs. The most common of these workgroups related to clinical governance, prescribing, public user involvement, needs assessment, and information technology. A total of 63 per cent of LHCCs had 
workgroups for one or more of 13 disease groups, with maternal and child health, and care of the elderly being the most prevalent workgroups. The development and planning of community, primary care, and out-of-hours services was carried out in separate workgroups in 25 per cent of LHCCs. Workgroups had been created for specific health care professionals such as practice managers, nurses, and professions allied to medicine in 17 per cent of LHCCs. A total of 8 per cent of LHCCs exhibited a workgroup to develop partnership working with other agencies.

\section{The mode of LHCC control}

The mode of control was assessed with respect to LHCC operation and decision making processes. Although LHCCs act as separate management entities, they are operational units within PCTs (Secretary of State for Health, 1997a). The extent to which LHCC development was controlled by PCT management as opposed to the LHCC itself was 40 per cent. Comments made by respondents indicated that the extent to which LHCC development was controlled by PCT management was expected to drop in the future.

The nature of the decision making process was investigated given that there is no blueprint for the management arrangements of LHCCs (NHSME, 1999). The extent to which LHCC managers were involved in decision making compared to GPs and other health and social care professionals was 40 per cent. Additionally, three percent of LHCCs had a management style based on central authority, whereas 25 per cent of LHCCs reported a fully participative management style.

LHCCs used a variety of formal and informal monitoring mechanisms to ensure compliance with their policies. These were "practice-based audit" (84 per cent of LHCCs), "communication by LHCC management" (82 per cent of LHCCs), "protocols and guidelines" (81 per cent of LHCCs), "representation on LHCC subgroups" (81 per cent of LHCCs), and "LHCC newsletters and mailings" (78 per cent of LHCCs). More voluntary instruments such as "peer pressure" (44 per cent of LHCCs), "goodwill of staff of participating practices" (44 per cent of LHCCs), and "performance management framework with standards and performance indicators" (44 per cent of LHCCs) proved less popular. LHCCs relied on an average of five out of the eight proposed monitoring mechanisms.

\section{Relationships among participating practices}

LHCCs represent a move away from an individual general practice model towards a collective arrangement, in which GPs and other health and social care professionals work together to shape and deliver local population-wide policies (Secretary of State for Health, 1997a).

Respondents quantified the relative importance of four types of partnership that can govern the relationship among participating practices on a scale of 0 per cent to 100 per cent (Pratt et al., 1998). The dominant type of partnership 
JHOM

17,1

was co-ordination, i.e. "general practices working together to deliver clear, common objectives" (average importance of 36 per cent). A co-ordinating partnership aims to bring together known good practice to achieve collective goals. The second most important type of partnership was co-evolution, "general practices work together in the presence of less clearly defined common objectives and uncertain ways to achieve those objectives" (average importance of 29 per cent), followed by co-operation, "general practices work together out of personal interest" (average importance of 22 per cent). Competition, "general practices compete with each other", was the least important type of partnership (average importance of 13 per cent). Only 18 per cent of LHCCs indicated that one type of partnership governed relationships within the LHCC. Hence, LHCCs need to be recognised as hybrid organisational forms characterised by elements of more than one type of partnership.

Once a general practice has joined an LHCC, it needs to determine whether and how much - it wishes to co-operate with other participating practices. LHCCs reported their agreement with a number of factors facilitating cooperation among participating practices on a five-point Likert scale. The highest level of agreement was attained with respect to the attitude of LHCC management and participating practices, i.e. "good leadership by LHCC management" (score of 4.41), "good working relationships between participating practices" (score of 4.34), and "enthusiastic GPs" (score of 4.28). This was followed by LHCC institutional arrangements, such as "effective organisation" (score of 4.22), "LHCC is seen by participating practices to achieve results" (score of 4.07), "strong involvement of participating practices in decision making" (score of 4.04), and "like-minded participating practices" (score of 3.89). LHCCs agreed less with benefits accruing to participating practices, including "perceived financial gain by participating practices" (score of 3.73) and "adequate financial, management, and human resources support" (score of 3.70), and a "good relationship with HB/PCT" (score of 3.54) as facilitators of co-operation among participating practices.

The level of agreement with a number of barriers to co-operation among participating practices was measured on a five-point Likert scale. "Extra time commitment of GPs" (score of 4.20) and "individualistic culture of GPs" (score of 3.86) received the highest level of agreement. This was followed by "lack of financial, management, human resources support" (score of 3.52), "differences among participating practices in the extent to which they wish to participate in the LHCC" (score of 3.46), "lack of independence from HB/PCT" (score of 3.33), and "poor relations with $\mathrm{HB} / \mathrm{PCT}$ " (score of 3.25). Less agreement was expressed with respect to other barriers such as "lack of government policy on LHCC development" (score of 3.22), "difficulties in communication between different health care professionals" (score of 3.06), and "lack of commitment of participating practices" (score of 2.95). 
LHCC management costs

The average management allowance amounted to $£ 166,613$ with a minimum of $£ 22,000$ and a maximum of $£ 400,000$. Management expenditure per capita ranged from $£ 1.14$ to $£ 5.14$, with an average of $£ 2.97$. This is comparable with management expenditure per capita of other types of integration in primary care such as first-wave total purchasing pilots (average of $£ 3.12$, adjusted to $2000 / 2001$ prices) and second-wave total purchasing pilots (average of $£ 2.65$, adjusted to 2000/2001 prices) (Posnett et al., 1998).

Management costs were generally not related to LHCC population and practice characteristics. The only exception was that the management allowance per practice was lower in LHCCs with more general practices (Pearson rank-order correlation coefficient $=-0.345 ; p=0.04$ ). There was no statistically significant relationship between the size of an LHCC (measured by the number of GPs, the number of WTE GPs, the number of general practices, and the patient population covered) and the management allowance per capita, although the sign of the effect was negative. This implies that the relationship between size and management costs is not straightforward, although the negative sign seems to indicate that, as LHCCs grow in size, managerial economies of scale may offset increasing costs of internal co-ordination.

\section{Discussion}

In the absence of central guidance, many aspects of the development and management of LHCCs have been devolved to individual LHCCs. This paper has presented a picture of heterogeneity among LHCCs in terms of the organisational structure of LHCCs, modes of control within LHCCs, relationships among participating practices, and management costs. The adaptation of policies to local circumstances is valuable as long as it does not affect the efficiency and equity of health care provision in Scotland.

Past studies largely related to the formation phase of LHCCs and were anecdotal and qualitative in nature (Hopton and Heaney, 1999; Goldie and Sheffield, 2000). Our results were based on a nationwide survey of Scottish LHCCs and measured various aspects of the development and management of LHCCs during their first year.

The following limitations of the study should be noted when interpreting the results. Although the response rate of 35 per cent was disappointing, some LHCC general managers declined to fill in our questionnaire due to time constraints or because they had already completed another questionnaire that was posted a couple of months earlier (Audit Scotland, 2001; LHCC Best Practice Group, 2001). Moreover, the use of closed questions limits the contextual detail that can be learned from respondents. Qualitative case studies are needed to gain a deeper understanding of the organisation and operation of LHCCs.
Integrated primary care 
$\mathrm{JHOM}$

17,1

\section{Implications for practice}

The results also have policy implications for the future development and management of LHCCs. LHCC management bodies relied on the commitment of a small group of representatives such as members of participating practices, nurses, and other health care professionals. The inclusion of mental health representatives, local health council representatives, voluntary service representatives, and lay representatives was marginal. This implies that stakeholder representation was not socially inclusive during the first year of LHCC operation. Attempts to engage patients and local communities had been limited and need to be stepped up to increase responsiveness and accountability to local health care users. Alternative ways to give a stronger voice to these other stakeholders in LHCC decision making need to be developed.

One of the main challenges facing LHCCs is to improve the quality and standards of clinical care within the constraints of a fixed budget. LHCCs tackled this priority by identifying clinical governance and prescribing as responsibilities of management bodies and by having workgroups set up specifically for this purpose. Moreover, LHCCs had introduced a variety of formal and informal monitoring mechanisms.

A number of factors were identified that influence the extent to which general practices are working together within LHCCs. Results on the factors facilitating co-operation among participating practices indicated that LHCCs need to focus on issues of leadership, organisation, and involvement in decision making to achieve their objectives. However, LHCCs also need to address factors such as GP workload, individualistic culture of GPs, and lack of LHCC support which impede co-operation among participating practices.

The extent to which LHCC development was controlled by PCT management was 40 per cent. A majority of LHCCs agreed that the lack of 
independence from the $\mathrm{HB} / \mathrm{PCT}$ acted as a barrier to co-operation among participating practices. However, LHCCs also wished to receive more financial, management, and human resources support. This seems to suggest that LHCCs view the role of the HB/PCT to consist of providing support activities, whilst they wish to be in charge of LHCC development and management.

The level of the management allowance per capita was not related in any systematic way to LHCC population and practice characteristics. For instance, the management allowance per capita was not affected by rurality or population morbidity and deprivation. This suggests that LHCCs with the greatest need do not necessarily get more resources to cater for their patient population, suggesting vertical inequity between LHCCs. The overall absence of any relationship between the management allowance per capita and LHCC population and practice characteristics points towards a lack of consistency in the process by which the management allowance is negotiated between the PCT and the LHCC. To date, no guidance has been issued with respect to supporting LHCC running costs. Moreover, the required level of investment in management is not known.

Finally, the results have implications for the size of LHCCs. There was wide variation in the population covered by LHCCs, which ranged from 1,000 to 160,000 people. The management allowance per practice was lower in LHCCs with more general practices. Ways of gaining some of the benefits of increased size without experiencing its disadvantages were to share support functions with the PCT or neighbouring LHCCs, and to create smaller locality groups within the LHCC with devolved responsibilities. However, more information is required about the costs and benefits of LHCCs of different sizes before LHCC reconfigurations or LHCC mergers can be considered.

\section{References}

Audit Scotland (2001), Paying dividends, Local healthcare co-operatives bulletin, Audit Scotland, Edinburgh.

Goldie, D. and Sheffield, J. (2000), "How do key stakeholders feel about the recent changes in general practitioner roles and relationships in the NHS?", paper presented at the 4th International Conference on Strategic Issues in Health Care Management, University of St Andrews, 30 March-1 April.

Grossman, S. and Hart, O. (1986), "The costs and benefits of ownership: a theory of vertical and lateral integration", Journal of Political Economy, Vol. 94, pp. 691-719.

Hopton, J. and Heaney, D. (1999), "Towards primary care groups: the development of local health care co-operatives in Scotland", British Medical Journal, Vol. 318, pp. 1185-7.

Kendall, M. (1975), Rank Correlation Methods, Griffin, London.

LHCC Best Practice Group (2001), Connecting communities with the NHS, Report of the LHCC Best Practice Group, Scottish Executive Health Department, Edinburgh.

NHS Management Executive (1999), LHCC development, NHS MEL(1999)13, Department of Health, Edinburgh. 
JHOM

17,1

36

Posnett, J., Goodwin, N., Griffiths, J., Killoran, A., Malbon, G., Mays, N., Place, M. and Street, A. (1998), "The transactions costs of total purchasing", National Evaluation of Total Purchasing Pilot Projects Working Paper, King's Fund, London.

Pratt, J., Plamping, D. and Gordon, P. (1998), "Partnership: fir for purpose?", Whole Systems Thinking Working Paper Series, King's Fund, London.

Regen, E., Smith, J. and Shapiro, J. (1999), "First off the starting blocks: lessons from GP commissioning pilots for PCGs", Health Services Management Centre, University of Birmingham, Birmingham.

Scottish Executive (2000), Our National Health: A Plan for Action, a Plan for Change, NHS in Scotland, Edinburgh.

Scottish Executive Health Department (2000), "Fair shares for all", final report of the national review of resource allocation for the NHS in Scotland, Scottish Executive, Edinburgh.

Secretary of State for Health (1997a), Designed to Care: Renewing the National Health Service in Scotland, Cm 3811, HMSO, London.

Secretary of State for Health (1997b), The New NHS: Modern and Dependable, HMSO, London (Cm 3807).

Simoens, S. and Scott, A. (1999), "Towards a definition and taxonomy of integration in primary care”, HERU discussion paper 03/1999, Health Economics Research Unit, University of Aberdeen, Aberdeen.

Smith, J., Regen, E., Goodwin, N., McLeod, H. and Shapiro, J. (2000), "Getting into their stride: interim report of a national evaluation of primary care groups", Health Services Management Centre, University of Birmingham, Birmingham. 